\title{
Control DE Un Sistema DE ENERGía BASADO EN VOLANTES DE INERCIA PARA MITIGAR LOS HUECOS DE TENSIÓN EN EL PUNTO DE CONEXIÓN COMÚN
}

\section{Control of a System of EnERGy BASED ON FlywheEl TO Mitigate the Voltage Gaps at the Point of Common Coupling}

\author{
Carlos Orellana Uguña ${ }^{1, *}$, Luis González Morales ${ }^{2}$, Nuno Abreu Sousa ${ }^{3}$
}

\section{Resumen}

Este artículo presenta el diseño de un sistema de energía basado en volante de inercia para mitigar los huecos de tensión. Con el sistema se mejora la calidad de energía en un punto de una red de distribución, el cual está expuesto a la conexión aleatoria de máquinas eléctricas. Para ello, se modela el sistema de distribución de energía, el sistema de inyección de energía que está compuesto por una máquina eléctrica con volante de inercia, el sistema de conversión de energía bidireccional y el sistema de control de corriente, voltaje y velocidad. El sistema diseñado permite inyectar una potencia de $22.8 \mathrm{~kW}$ y capacidad de $1.2 \mathrm{Wh}$, compensando los transitorios producidos por las cargas conectadas a la red.

Palabras clave: baterías, volante de inercia, $\mathrm{AC} / \mathrm{DC}, \mathrm{DC} / \mathrm{AC}, \mathrm{PCC}$

\section{Abstract}

This article presents the design of a power system based on flywheel to mitigate voltage sags. With this system the power quality is improved at a point in a distribution network, which is subject to the random connection of electric machines. For this purpose, the power distribution system is modeled, the power supply system which is composed of an electric machine with flywheel, the bidirectional energy conversion system and the current, voltage and speed control system. The designed system enables supplying a power of $22.8 \mathrm{~kW}$ and capacity of $1.2 \mathrm{Wh}$, compensating the transients produced by the loads connected to the network.

Keywords: Batteries, flywheel, AC/DC, DC/AC, PCC.

\footnotetext{
$\overline{1, *}$ Instituto Tecnológico Superior Luis Rogerio González, Azogues - Ecuador.

Autor para correspondencia : carl.mauryou@yahoo.es. (D) http://orcid.org/0000-0001-7214-4060

${ }^{2}$ Departamento de Ingeniería Eléctrica Electrónica y Telecomunicaciones (DEET), Universidad de Cuenca, Cuenca Ecuador. (D) http://orcid.org/0000-0001-9992-3494

${ }^{3}$ Departamento de Engenharia Electrotécnica da Escola Superior de Tecnologia e Gestãodo Instituto Politécnico de Leiria - Portugal. (D) http://orcid.org/0000-0002-3837-0547
}

Recibido: 22-01-2020, aprobado tras revisión: 19-05-2020

Forma sugerida de citación: Orellana Uguña, C.; González Morales, L. y Abreu Sousa, N. (2020). «Control de un sistema de energía basado en volantes de inercia para mitigar los huecos de tensión en el punto de conexión común». INGENIUS. N. ${ }^{\circ}$ 24, (julio-diciembre). pp. 49-58. DOI: https://doi.org/10.17163/ings.n24.2020.05. 


\section{Introducción}

La empresa distribuidora de energía eléctrica, al ser la entidad que suministra energía a los consumidores, basándose en normas establecidas, tiene la obligación de mantener la calidad de energía entregada, la que se puede estimar mediante la continuidad de servicio y la calidad de la onda de tensión.

Para llevar a cabo este objetivo se implementaron diferentes estrategias de respaldo de energía en los sistemas eléctricos de potencia, las cuales buscan mejorar la calidad y estabilidad de la energía [1]. Estos sistemas pueden ser clasificados por su tiempo de descarga [2] como se indica a continuación:

Tiempo de descarga de segundos a minutos: son usados para mejorar la calidad de energía. Los tiempos de descarga son de 10 minutos con respuestas de milisegundos. En este tipo se encuentran los supercondensadores con potencias de hasta $1 \mathrm{MW}$ y los volantes de inercia con potencias entre $10 \mathrm{~kW}$ y $1 \mathrm{MW}$.

Tiempo de descarga de minutos a una hora: son usados como puentes de potencia, es decir, aseguran la confiabilidad de la fuente de alimentación a los clientes, poseen respuestas de segundos a minutos con tiempos de descargas de hasta 1 hora, con potencias entre $1 \mathrm{~kW}$ y $10 \mathrm{MW}$. En este tipo figuran las baterías electroquímicas.

Tiempo de descarga de horas: se utiliza para gestión de la energía, en este tipo se encuentra el almacenamiento hidráulico por bombeo y por aire comprimido, con potencias entre $100 \mathrm{MW}$ y $1 \mathrm{GW}$ respectivamente y el almacenamiento de energía térmica con capacidades de almacenamiento entre $10 \mathrm{MW}$ y $100 \mathrm{MW}$.

Independientemente de la tecnología utilizada se debe considerar aspectos como: gastos operativos y constructivos, tiempo de vida útil, tiempo de respuesta ante una perturbación en la red, limitaciones geográficas y propiedades físicas del mismo [3].

Si se consideran las limitaciones geográficas se descartan los sistemas de almacenamiento por bombeo, pues requieren de dos depósitos que se encuentren a diferentes niveles, debido a que la energía almacenada es proporcional al volumen del líquido y a la diferencia de altura entre los depósitos, limitando así su instalación solo a lugares con características no planas. No se opta por el aire comprimido debido a que requiere cavernas subterráneas con características muy resistentes, pues albergan aire a gran presión y, finalmente, se descarta el almacenamiento de energía térmica debido a que el principio de funcionamiento de cada uno de ellos depende de la temperatura ambiente en la cual va a trabajar.
Como resultado quedan las baterías electroquímicas, los volantes de inercia y los supercondensadores [2].

Las baterías electroquímicas presentan desventajas, tales como: al finalizar su vida útil y al no poseer una infraestructura adecuada de reciclaje contamina el medioambiente [4], su vida útil depende del ambiente de trabajo y del número de cargas/descargas $\mathrm{y}$, lo más importante, no pueden cargarse ni descargarse rápidamente debido a que poseen una resistencia interna grande [5]. Debido a ello queda como resultado la utilización de los volantes de inercia que presentan las siguientes ventajas: tienen un mayor ciclo de cargas/descargas con potencia media a alta (kW a MW) durante cortos periodos (segundos) sin afectar su vida útil [6], alta capacidad de respuesta, es amigable con el ambiente pues no requiere de reacciones químicas, no necesita de condiciones geográficas especiales para su construcción [7]. El principal competidor en términos comparativos que tienen los volantes de inercia son los supercondensadores, los cuales de igual manera presentan las siguientes ventajas sobre las baterías electroquímicas: pueden cargarse/descargarse en períodos cortos (segundos), pueden proporcionar corrientes de cargas altas, poseen un ciclo de vida del orden de millones de veces, trabajan en condiciones de temperaturas muy duras, no poseen en su estructura elementos tóxicos [2].

En este trabajo se pretende realizar el estudio sobre el diseño de un sistema de inyección de energía basado en volantes de inercia para mitigar los huecos de tensión en el punto de conexión común de un sistema eléctrico de distribución. Para ello se modelará el volante de inercia con su sistema de control y la red de distribución. El diseño es aplicado para una zona rural con explotación minera y agrícola que presenta cargas del tipo máquinas eléctricas, usando el PSIM ${ }^{\circledR}$ como herramienta de simulación.

\subsection{Descripción del problema}

Debido al incremento de cargas conectadas al punto de conexión común (PCC) perteneciente a la zona con vocación minera y agrícola de estudio, la calidad del suministro de energía disminuye, por las características del sistema de distribución existente y la continua puesta en marcha de las máquinas eléctricas de gran capacidad, sin contar con los arrancadores suaves que contribuyen a la formación de huecos de tensión. El caso de estudio abarcará al cantón Pucará y sus alrededores, específicamente el caserío San Juan de Naranjillas perteneciente a la provincia del Azuay, Ecuador, la cual es abastecida por el alimentador 1424 de la Empresa Eléctrica Regional Centro Sur.

Debido a la falta de existencia de normativas técnicas que regulen la potencia de los motores utilizados en las diferentes actividades mineras/agrícolas, se consideró como referencia las maquinarias utilizadas en una 
principal minera denomina TRES CHORRERAS, que establece la utilización de motores Allis Chalmers de $32 \mathrm{HP}$, en función de ello y según catálogos disponibles en el Ecuador se utiliza motores eléctricos W21 a Prueba de Explosión NEMA Premium Efficiency de $50 \mathrm{HP}$ que dan movimiento a bombas centrífugas para el manejo de líquidos, trituradoras, cintas transportadoras entre otras [8].

Las características de los motores a utilizar se indican en la Figura 1 [9].

El modelo de simulación para la red de distribución se indica en la Figura 1, con resistencias de $20 \Omega$ que emulan pequeños aparatos eléctricos distribuidos en cada una de las fases (iluminación, electrodomésticos).

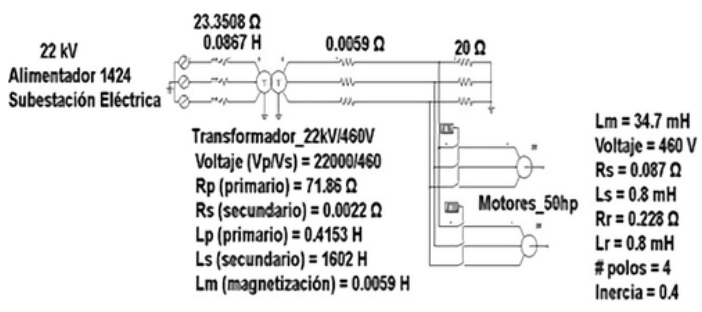

Figura 1. Modelo de la red de distribución y características de los motores de $50 \mathrm{Hp}$

\subsection{Calidad de energía}

Uno de los aspectos importantes relacionados con la calidad de energía son los llamados huecos de tensión sag descritos en detalle en las normativas técnicas IEEE 1159, CONELEC 004/01 y NP EN 50160-2010, las cuales lo definen como una disminución de la tensión de alimentación a un valor situado entre el $10 \%$ y el $90 \%$ de la tensión nominal. Además, las normas definen a la elevación de tensión swell como una elevación de tensión de alimentación a un valor situado entre el $110 \%$ y $180 \%$ de la tensión nominal, seguida del restablecimiento de la tensión después de un corto lapso de tiempo. Aplicando este criterio al nivel de tensión fase-neutro del lado secundario del transformador de la Figura 1, el rango para la formación de los sags está comprendido entre $26,55 \mathrm{~V}$ y $238,95 \mathrm{~V}$ y para el swell entre $292,05 \mathrm{~V}$ y $477,9 \mathrm{~V}$, resultando así un rango permisible de $238,95 \mathrm{~V}$ hasta $292,05 \mathrm{~V}$.

A continuación, se describirán los métodos utilizados en este trabajo de investigación para mitigar la formación de los huecos de tensión.

\section{Materiales y métodos}

\subsection{Propuesta del sistema de energía para mitigar los huecos de tensión en el punto de conexión común}

El modelo en diagrama de bloques que este estudio propone para mitigar los huecos de tensión en el PCC está indicado en la Figura 2, el cual está compuesto por una máquina eléctrica con volante de inercia, dos convertidores trifásicos de potencia controlados y un condensador de acoplamiento denominado DC-link.

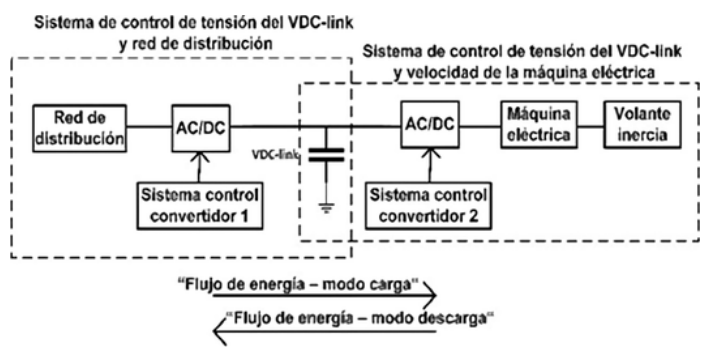

Figura 2. Sistema basado en volante de inercia para mitigar los huecos de tensión en el PCC

En la Figura 2, el modelo propuesto consta de las siguientes etapas de control:

Sistema de control 1: el convertidor de potencia asociado a la red de distribución consta de un control de corriente en el sistema de referencia síncrono como el utilizado en [10], [11]. Su tarea es controlar la tensión de la red de distribución y la tensión DC-link. Este control se puede dividir en dos subetapas: modo carga, la energía fluye desde la red de distribución hacia el bus de continua para alcanzar el nivel de tensión nominal del $V_{D C-\text {-link }}$. En este modo, opera el sistema de control de tensión del $V_{D C-\text {-link }}$ usando al convertidor como un rectificador trifásico controlado. En modo descarga, la energía fluye desde el bus de continua hacia la red distribución, en este modo, opera el sistema de control de tensión de la red de distribución usando al convertidor como un inversor trifásico controlado.

Sistema de control 2: el convertidor de potencia asociado a la máquina eléctrica consta de un control de corriente en el sistema de referencia síncrono. Este sistema de control se puede dividir en dos subetapas: modo carga, la energía fluye desde la fuente de tensión $V_{D C-l i n k}$ hacia la máquina eléctrica que está funcionando como motor para alcanzar la velocidad nominal de carga, en este modo, opera el sistema de control de velocidad de la máquina eléctrica usando al convertidor como un inversor trifásico controlado. En este estado, el sistema tiene almacenada su energía en forma cinética. En modo descarga, la energía fluye desde la máquina eléctrica funcionando como generador hacia la fuente $V_{D C-l i n k}$ para mantener su nivel de tensión en un valor de referencia ante la presencia de cualquier carga conectada a la red de distribución. En este modo opera el sistema de control de tensión del circuito intermedio $V_{D C-l i n k}$, usando al convertidor como un rectificador trifásico controlado. 
El cambio de los modos de control está sujeto al estado de la tensión en el PCC; en este sentido, cada uno de los convertidores de potencia operará de forma $\mathrm{AC} / \mathrm{DC}$ o $\mathrm{DC} / \mathrm{AC}$.

A continuación, se describen los lazos de control que conforman el sistema de inyección de energía basado en volante de inercia.

\subsubsection{Sistema de control 1 , perteneciente al control de la tensión de la red de dis- tribución y tensión del $V_{D C-l i n k}$.}

El sistema de control 1, cuenta con una estructura definida por el modo de operación carga o descarga. La Figura 3 indica en forma de diagrama de bloques el sistema de control en cascada.

Por el nivel de tensión que soportan el convertidor trifásico SKiM459GD12E4 y el condensador de acoplamiento U37F tipo E37F501CPN103MFK0M, se establece como referencia un nivel de tensión de 900 Vcd para el $V_{D C-l i n k}$.

\section{MODO CARGA - MÁQUINA SINNCRONA COMO MOTOR}

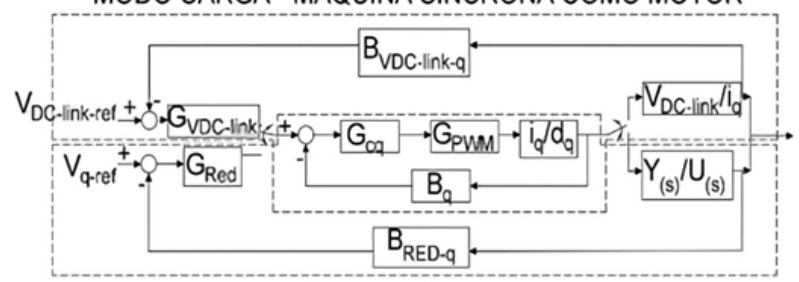

MODO DESCARGA - MÁQUINA SÍNCRONA COMO GENERADOR

Figura 3. Lazos de control para la tensión de la red de distribución y del $V_{D C-l i n k}$

De la Figura $3, B_{q}, B_{R e d-q}, B_{V D C-l i n k}$ son las ganancias asociadas a las mediciones de corriente, de tensión de la red de distribución y de la tensión del $V_{D C-\text { link }}$ respectivamente con valores unitarios, $G_{c q}$, $G_{V D C-l i n k}, G_{R E D}$ son las funciones de transferencia de los controladores, que en esta aplicación se han utilizado controladores clásicos de configuración proporcional e integral (PI), $G_{P W M}$ es la ganancia del PWM, que en este caso se ha utilizado con ganancia unitaria, $\frac{Y(s)}{U(s)}$ representa la función de transferencia que relaciona la tensión de la red de distribución con respecto a la corriente en el eje de cuadratura, $\frac{V_{D C_{l} i n k}}{\tilde{i}_{q}}$ constituye la función de transferencia que relaciona la tensión del $V_{D C-l i n k}$ con respecto a la corriente en el eje de cuadratura.

El modelado del convertidor de potencia puede tratarse mediante la Ecuación (1), como es utilizada en [10], [11].

$$
\frac{\tilde{i}_{d(s)}^{\prime}}{\tilde{d}_{d(s)}^{\prime}}=\frac{\tilde{i}_{q(s)}^{\prime}}{\tilde{d}_{q(s)}^{\prime}}=\frac{V_{0}}{L s^{\prime}+R_{s}}
$$

Para los parámetros involucrados en la Ecuación (1), se considera lo siguiente:
$V_{0}$ es la tensión del $V_{D C-l i n k}$, en el presente trabajo se ha definido en un valor de $900 \mathrm{~V}$.

$L$ es la inductancia del filtro de entrada de acuerdo con [12]. Para el cálculo se procede de la siguiente manera: se considera que la reactancia inductiva es igual al $10 \%$ de la resistencia base [10], resultando entonces $X l=0,565 \Omega$, por tanto, $L=1,5 \mathrm{mH}$. A partir de este valor inicial se calibra el valor del filtro $L$ hasta llegar a un valor de $7 m H$, con el cual la distorsión armónica total (THD, por sus siglas en inglés) se encuentra dentro de la norma NP EN 50160-2010, Conelec 004/01 (menor al $8 \%$ ).

$R_{s}$ de acuerdo con [12] está asociada a las pérdidas de los elementos pasivos y semiconductores que existe en el convertidor.

$s^{\prime}$ es el operador de Laplace.

De igual manera, se cumplen las ecuaciones del balance de energía asociada al condensador del bus de continua que puede representarse mediante las Ecuaciones $(2)$ y (3) [10].

$$
\frac{V_{D C \_l i n k}}{\tilde{i}_{q}}=\frac{D_{q}}{C s^{\prime}+\frac{I_{0}}{V_{D C \_l i n k}}}
$$

$$
D_{q}=\frac{2 \sqrt{2} V_{f-n}}{V_{D C \_l i n k}}
$$

Dónde: $D_{q}$ es el índice de modulación, $C$ es la capacitancia total de los condensadores que conforman el bus de continua, $I_{0}$ es la corriente que circula desde el puente rectificador hacia el bus de continua, $V_{D C_{l} i n k}$ es la tensión del bus de continua, $s^{\prime}$ es el operador de Laplace.

En el caso del modo descarga, el convertidor de potencia controlado mediante los lazos de corriente en el eje directo y cuadratura es utilizado para el control de la tensión en la red de distribución mediante la función de transferencia $\left(\frac{Y(s)}{U(s)}\right)$ que para efecto del diseño del sistema de control se ha caracterizado a una aproximación de un sistema de primer orden. Para encontrar los parámetros que constituyen dicha función de transferencia se considera que el sistema basado en el volante de inercia va a estar conectado en paralelo a la red de distribución comportándose como fuente de corriente. De forma gráfica, la Figura 4 muestra el esquema para determinar la función de trasferencia antes descrita mediante la respuesta escalón. 


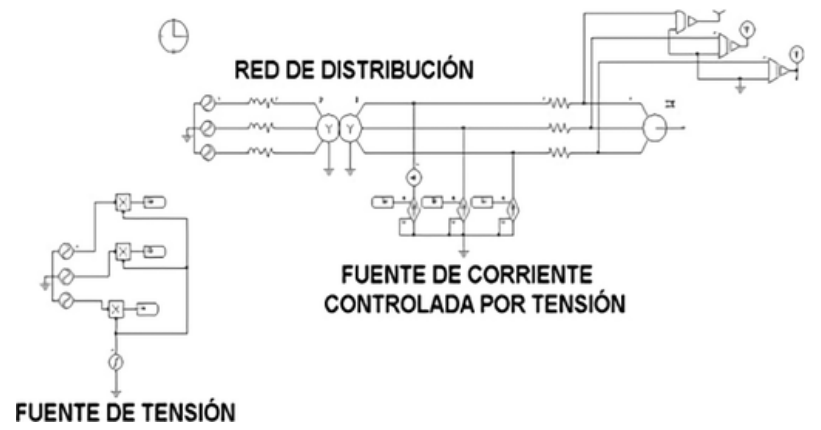

Figura 4. Modelo para la función de transferencia que relaciona la tensión en el PCC con la inyección de corriente en fase

$\mathrm{Al}$ aplicar el procedimiento antes descrito, el comportamiento de la tensión en la red de distribución ante una perturbación de corriente con amplitud de $\Delta I=400 \mathrm{~A}$, resulta en $\Delta V=51,6 \mathrm{~V}$, obteniendo una función de transferencia como la que observa en la Ecuación (4).

$$
\frac{Y_{(s)}}{U_{(s)}}=\frac{0,129}{0,287 s^{\prime}+1}
$$

Es importante destacar que las corrientes que se inyecten desde el sistema de almacenamiento de energía hacia el PCC deben tener un sistema de sincronización de fase para alcanzar el control de la energía activa o reactiva. En este diseño se ha utilizado un sistema de enganche de fase PLL normalizado como el utilizado en [10].

\subsubsection{Sistema de control 2 , perteneciente al control de la velocidad de la máquina eléctrica y la tensión del $V_{D C-l i n k}$.}

La máquina eléctrica utilizada en el presente trabajo es una máquina síncrona de imanes permanentes debido a que presenta algunas ventajas sobre las de inducción, entre las cuales están: alta eficiencia, excelente densidad de potencia, buena relación par mecánico/corriente, tamaño pequeño [13]. Lo que se destaca en las máquinas síncronas es que la velocidad del rotor es igual a la velocidad del flujo del rotor. Por tanto, $\theta_{1}$ (ángulo necesario para la transformada de Park) se mide directamente mediante sensores de posición o mediante la integración de la velocidad del rotor [14].

Para el control de la velocidad de la máquina síncrona se utilizó el control de campo orientado, FOC, el cual permite desacoplar el par mecánico y las componentes de flujo de magnetización, debido a la facilidad de poder controlar de forma independiente la corriente $i_{s d}$ (asociada al flujo magnético de la máquina) e $i_{s q}$ (asociada al par mecánico ejercida por la máquina), esto gracias a su característica de rotor liso $\left(L_{d}=L_{q}\right)[10]$.
El modelo matemático de la máquina síncrona de imanes permanentes en el sistema de referencia síncrono es expresado por las Ecuaciones (5) y (6).

$$
\begin{gathered}
V_{d}=R_{s} I_{d}-w L_{q} I_{q}+L_{d} \frac{d l_{q}}{d t} \\
V_{q} w L_{d} I_{d}+R_{s} I_{q}+L_{q} \frac{d I_{q}}{d t}+\lambda_{m} \frac{\sqrt{6}}{2} w
\end{gathered}
$$

Donde: $w$ es la velocidad angular eléctrica en $(\mathrm{rad} / \mathrm{s}) ; \lambda_{m}$ es el flujo de los imanes permanentes, $L_{q}$ y $L_{d}$ son las inductancias de la máquina en el eje síncrono de cuadratura y directo, respectivamente en $(\mathrm{H})$; $R_{s}$ es la resistencia estatórica de la máquina síncrona en $(\Omega)$.

La potencia efectiva y el par son expresados por las Ecuaciones (7) y (8).

$$
\begin{aligned}
& P_{e}=w\left[\left(L_{d}-L_{q}\right) I_{q} I_{d}+\lambda_{m} I_{q} \frac{\sqrt{6}}{2}\right] \\
& T_{e}=p\left[\left(L_{d}-L_{q}\right) I_{q} I_{d}+\lambda_{m} I_{q} \frac{\sqrt{6}}{2}\right]
\end{aligned}
$$

Dónde: $p$ es el número de polos de la máquina síncrona.

La relación que existe entre la velocidad angular del rotor y la corriente referida al eje de cuadratura, es expresada por la Ecuación (9).

$$
\frac{d w_{r}}{d t}+B w_{r}=p \lambda_{m} I_{q} \frac{\sqrt{6}}{2}
$$

Donde: $J$ es el momento de inercia en $\left(k g \cdot \mathrm{m}^{2}\right)$, B es el coeficiente de fricción en $(N \cdot m \cdot s)$ y $w_{r}$ es la velocidad angular mecánica.

La relación de la corriente en estado estable en el eje de cuadratura y la tensión del condensador del bus de continua se relacionan mediante la Ecuación (10).

$$
I_{q s}=\frac{V_{D C \_l i n k} I_{d c}}{p \lambda_{m} \frac{\sqrt{6}}{2} w_{r}}
$$

A partir de las Ecuaciones de la máquina síncrona juntamente con el condensador referidas al eje síncrono, se obtiene el diagrama de bloques de la Figura 5, en donde se observa un lazo interno perteneciente al lazo de corriente en el eje de cuadratura y dos lazos externos que corresponden al control de velocidad de la máquina y el control de la tensión del $V_{D C-l i n k}[15]$. 


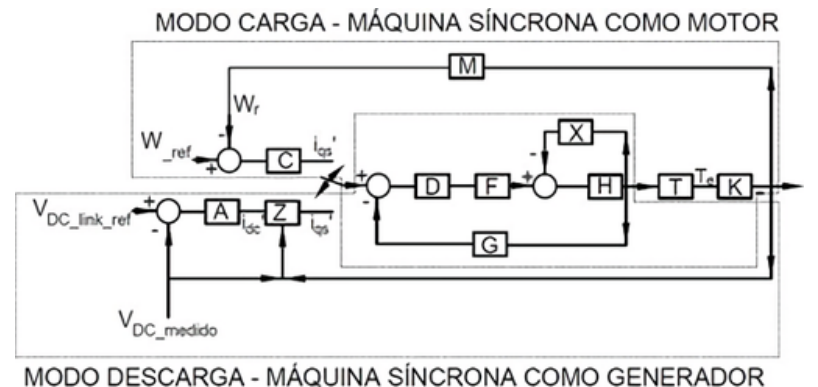

Figura 5. Lazo de control de la tensión del $V_{D C-l i n k}$ y velocidad de la máquina síncrona

De la Figura 5, G y M son las ganancias asociadas a los censados de corriente y de velocidad con valores unitarios, D, A, C son las funciones de transferencia de los controladores. En esta aplicación se ha utilizado controladores PI; F es la ganancia del PWM, que en este caso se ha utilizado con ganancia unitaria. $\mathrm{H}$ viene expresada por la Ecuación (1), $Z=\frac{2 V_{D C \text { medido } I_{d c}}}{p \lambda_{m} \sqrt{6} w_{r}}$, $T=\frac{p \lambda_{m} \sqrt{6}}{2}, X=\left(\frac{p \lambda_{m} \sqrt{6}}{2}\right)^{2}\left(\frac{1}{J s^{\prime}+B}\right), K=\frac{1}{J s^{\prime}+B}$.

Para la sintonización del lazo de velocidad y de corriente es necesario conocer los parámetros eléctricos y mecánicos de la máquina síncrona trifásica, estos datos están indicados en la Tabla 1 [15].

Tabla 1. Parámetros de la máquina síncrona de imanes permanentes

\begin{tabular}{lc}
\hline Potencia $(\mathrm{kW})$ & $<125$ \\
Polos & 2 \\
Velocidad $(\mathrm{krpm} / \mathrm{min})$ & $<36$ \\
Resistencia del estator $(\Omega)$ & 0,02 \\
Inductancia del estator $(\mu \mathrm{H})$ & entre 70 y 120 \\
Inercia $\left(\mathrm{kg} \cdot \mathrm{m}^{2}\right)$ & 0,633 \\
Coeficiente de fricción $(\mathrm{N} \cdot \mathrm{m} \cdot \mathrm{s})$ & $4,2 \times 10^{-05}$ \\
Inductancia externa $(\mathrm{mH})$ & 1,5 \\
\hline
\end{tabular}

La función de transferencia necesaria para la sintonización del controlador PI relacionado al lazo de control de tensión del $V_{D C-l i n k}$ es la misma que se expresa en las Ecuaciones (2) y (3).

Las Ecuaciones (11) y (12) son el resultado de la simplificación de los lazos de corriente y de velocidad de la máquina síncrona.

$$
\frac{i_{q s}}{i_{q s}^{\prime}}=\frac{E s^{\prime 2}+P s^{\prime}+N}{Q s^{\prime 3}+I s^{\prime 2}+Y s^{\prime}+U}
$$

Donde:

$$
\begin{aligned}
& E=4 J L V_{D C-l i n k}, P=\left(4 L B V_{D C-l i n k}+\right. \\
& \left.4 J R V_{D C-l i n k}\right) . \\
& N=4 R B, L=L_{\text {máquina-síncrona }}+L_{\text {externa }} \\
& \text { pertenecientes al numerador de la Ecuación }(11) . \\
& Q=4 J L^{2}, I=4 L^{2} B+8 J R L, Y=8 R B L+ \\
& 6 V_{D C-l i n k} p^{2} \lambda_{m}^{2} L, U=4 R^{2} B+6 V_{D C-l i n k} p^{2} \lambda_{m}^{2} R
\end{aligned}
$$

$L=L_{\text {máquina-síncrona }}+L_{\text {externa }}$ pertenecientes al denominador de la Ecuación (11).

$$
\frac{w_{r}}{i_{q s}}=\frac{1,22 p \lambda_{m}}{J s^{\prime}+B}
$$

La sintonización de los PI para los diferentes lazos de control se realiza por asignación de polos y ceros, considerando los siguientes aspectos [11]:

La frecuencia de conmutación en este trabajo se ha fijado en $15 \mathrm{kHz}$, lo que limita las dinámicas de los lazos de corriente, dado que para frecuencias superiores a $\frac{f_{s w}}{2}=7.5 \mathrm{kHz}$, la técnica del modelado pierde validez.

A parir de la frecuencia de sintonización del PI para el lazo de corriente, se debe sintonizar una década menos para los lazos restantes de control.

El margen de fase del diagrama de Bode tiene que ser mayor o igual a $60^{\circ}$ eléctricos.

El margen de ganancia mayor o igual a $7 \mathrm{~dB}$.

Considerando estos aspectos se obtuvieron los siguientes parámetros del PI:

Sistema de control 1: para el lazo de corrientes en el eje directo y cuadratura el controlador PI posee un $k_{p}=0,36$ y $k_{i}=0,09$, para el lazo de control de tensión del bus de continua $V_{D C-\text { link }}$ el controlador PI posee un $k_{p}=51,02$ y $k_{i}=0,32$ y para el lazo de control de tensión de la red de distribución el controlador PI posee un $k_{p}=36,98$ y $k_{i}=4,3$.

Sistema de control 2: para el lazo de corrientes en el eje directo y cuadratura el controlador PI posee un $k_{p}=655$ y $k_{i}=166,62$, el lazo de control de tensión del bus de continua $V_{D C-l i n k}$ posee los mismos valores que del sistema de control 1 y para el lazo de control de la velocidad de la máquina síncrona el controlador PI posee un $k_{p}=9,5$ y $k_{i}=0,005$.

El sistema basado en el volante de inercia posee dos restricciones en modo descarga:

Nivel de tensión mínimo que puede tener el bus de continua $V_{D C-l i n k}$, que se calcula mediante la Ecuación (13) [10].

Para este trabajo, la tensión mínima es de $V_{d c_{m} \text { inimo }}=639 \mathrm{~V}$.

Profundidad de descarga de los volantes de inercia.

El cual se calcula como el $75 \%$ de su energía almacenada, que para este caso es de $w_{r-\text { minimo }}=$ $27 \mathrm{rad} / \mathrm{s}$. 


$$
V_{D C \_ \text {minimo }}=\frac{2 \sqrt{2} V_{f-n(r m s)}}{D_{q}}
$$

Si el sistema basado en volantes de inercia se encuentra por debajo de las restricciones en modo descarga, la referencia de corriente se fijará a un valor 0 , para que de este modo no inyecte ni absorba energía durante la presencia de un hueco de tensión en el PCC.

\section{Resultados y discusión}

\subsection{Desempeño de sistema de control}

Para comprobar el desempeño del sistema basado en volante de inercia se considera los límites establecidos en la sección 2, a partir de ello se procede a simular el modelo de la Figura 1, sin incluir el sistema basado en volante de inercia. El resultado del nivel de tensión está indicado en la Figura 6.

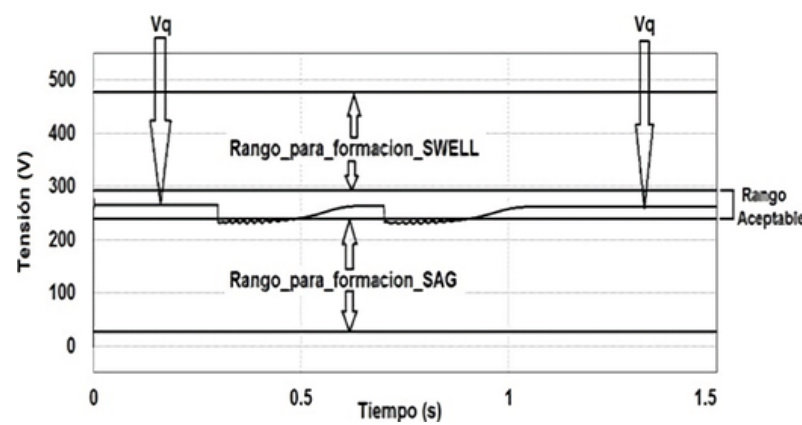

Figura 6. Comportamiento de tensión en el PCC al momento del arranque de los motores en la red de distribución sin el sistema de energía basado en volantes de inercia

$\mathrm{Al}$ observar la Figura 6, antes de ingresar en funcionamiento las cargas $(\mathrm{t}<0,3 \mathrm{~s})$, el nivel de tensión en el PCC es de $265,5 \mathrm{~V}$ encontrándose dentro de los márgenes aceptables establecidos en la sección 2 y con una corriente de $13 \mathrm{~A}$ (Figura 7) debido a la carga de $20 \Omega$ que se encuentra conectada.

$\mathrm{Al}$ momento de ingresar a funcionar el primer motor eléctrico $(\mathrm{t} \geq 0,3 \mathrm{~s})$ se observa una disminución repentina y transitoria de la tensión, con un valor igual a $231 \mathrm{~V}$ (Figura 6), el cual demanda un pico de corriente de $400 \mathrm{~A}$ con un tiempo de duración de $0.2 \mathrm{~s}$ indicado en la Figura 7. En esta condición, la tensión existente en el PCC se ubica dentro del rango establecido para la formación de un hueco de tensión (sags).

El tiempo en que ingresa nuevamente el nivel de tensión dentro del rango aceptable es de 0,2 s (Figura $6)$.

Tal comportamiento en $0,7 \mathrm{~s}$, debido a que se conecta el segundo motor, con el mismo valor de pico de corriente y tiempo de estabilización.

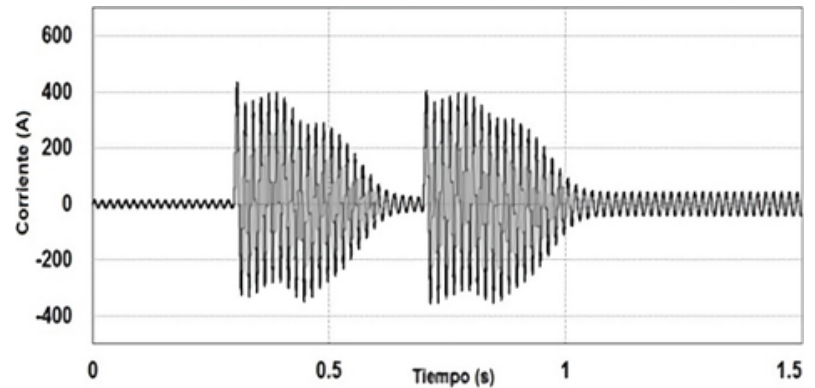

Figura 7. Comportamiento de corriente en la fase «A» En PCC al momento del arranque de la máquina eléctrica en la red de distribución sin el sistema de energía basado en volantes de inercia

Ahora se considera la conexión del sistema de energía basado en volantes de inercia, que inyecta energía para mitigar los huecos de tensión en el PCC obteniendo la Figura 8.

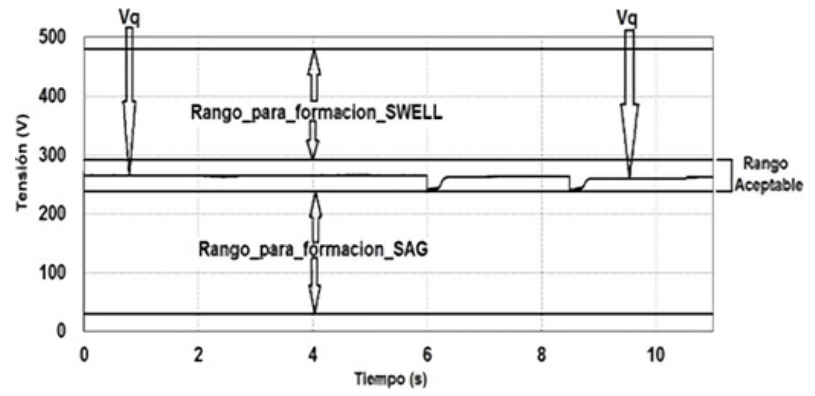

Figura 8. Nivel de tensión con la inclusión del sistema de energía basado en volantes de inercia para mitigar los huecos de tensión en el PCC

En la Figura 8 se puede observar que al momento del arranque del primer motor que ocurre en el tiempo de $6 \mathrm{~s}$, el nivel de tensión se encuentra dentro del rango aceptable establecido en la sección 2 , con un valor de $244 \mathrm{~V}$, en comparación con el nivel de tensión de la Figura 6 se incrementó $13 \mathrm{~V}$. De igual modo sucede en $8,5 \mathrm{~s}$ cuando ingresa a funcionar el segundo motor con nivel de tensión al momento del arranque de $244 \mathrm{~V}$.

En la Figura 9 se puede apreciar el nivel de tensión del $V_{D C-l i n k}$ y la velocidad de la máquina síncrona. Se observan los siguientes sucesos: antes de ingresar a funcionar los motores el nivel de tensión del $V_{D C-l i n k}$ se incrementa hasta un valor de referencia de $900 \mathrm{~V}$, con el convertidor de potencia asociado funcionando como rectificador para que de esta manera pueda absorber energía desde la red de distribución, con su respectivo lazo de corriente con referencia negativa igual a -20 A (Figura 10a). Cabe destacar que en esta condición, la corriente del sistema basado en volante de inercia denominada $I_{\text {filtro }}$ está desfasada de la corriente que circula a la carga (Figura 10a). De igual manera, en la Figura 9 se observa que la velocidad de la máquina síncrona empieza a incrementarse hasta un valor de referencia de $200 \mathrm{rad} / \mathrm{s}$ con el convertidor de potencia 
asociado funcionando como inversor, de esta manera, el volante de inercia absorbe energía desde la fuente de tensión del $V_{D C-l i n k}$, con su respectivo lazo de corriente con referencia positiva igual a $40 \mathrm{~A}$ (Figura 11). En este estado el sistema de energía basado en volante de inercia se encuentra en modo de carga.

Cuando la máquina síncrona funcionando como motor alcanza la velocidad de referencia respectiva consume una corriente total de 3,8 A (Figura 11). En este estado el sistema de energía basado en volante de inercia se encuentra en modo stand-by.

En el tiempo $t \geq 6$ s de la Figura 9 se observa una repentina disminución de la tensión del $V_{D C-l i n k}$ con un valor igual a $860 \mathrm{~V}$, esto se debe a que ingresó a funcionar el primer motor conectado al PCC, en este instante el convertidor de potencia asociado funciona como inversor para inyectar energía hacia la red de distribución, con su lazo de corriente con referencia positiva igual a $115 \mathrm{~A}$ (Figura 10b). Un aspecto importante es que la corriente del sistema basado en volante de inercia denominado $I_{\text {Filtro }}$ inyectada hacia la red de distribución se encuentra en fase con la corriente que circula hacia la carga (Figura 10b).

Para que la tensión del $V_{D C-l i n k}$ no disminuya bruscamente, la máquina síncrona empieza a funcionar como generador disminuyendo su velocidad hasta un valor de 164,3 $\mathrm{rad} / \mathrm{s}$ entregando energía mediante el convertidor de potencia en modo rectificador hacia la fuente de tensión del $V_{D C-l i n k}$, con su respectivo lazo de corriente con referencia negativa igual a -100 A (Figura 11).

La disminución de tensión del $V_{D C-l i n k}$ y la velocidad de la máquina se mantienen durante el arranque del motor que es de $0,2 \mathrm{~s}$. En este estado, el sistema basado en volante de inercia se encuentra en modo descarga.

Una vez transcurrido el tiempo de arranque del motor y cuando la tensión del PCC se encuentre dentro del rango establecido en la sección 3, la tensión del $V_{D C-l i n k}$ juntamente con la velocidad de la máquina se vuelven a incrementar hasta su valor de referencia (Figura 9), repitiéndose nuevamente el ciclo mencionado anteriormente cuando exista la presencia de otro hueco de tensión.

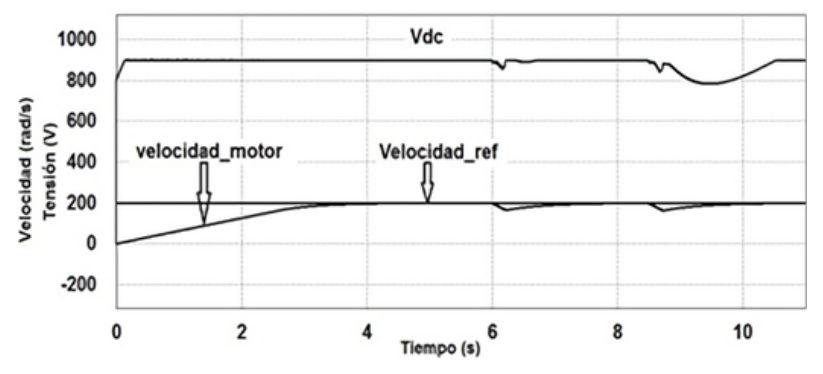

Figura 9. Nivel de tensión del $V_{D C-l i n k}$ y velocidad de la máquina síncrona
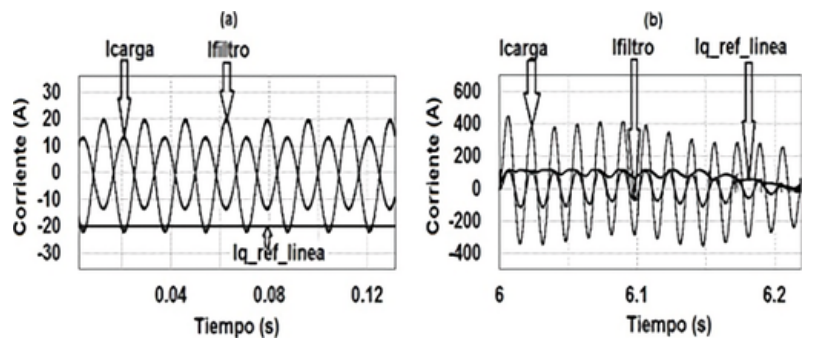

Figura 10. Corriente de referencia del sistema de energía basado en volantes de inercia: a) modo carga, b) modo descarga

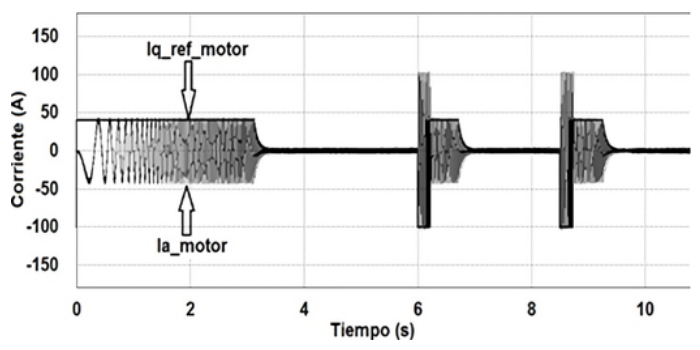

Figura 11. Corriente de referencia para la máquina síncrona

Otro aspecto importante es el nivel de THD que se produce al momento de carga, stand-by y descarga del sistema basado en volante de inercia. Tras el ensayo de simulación, se obtuvieron los siguientes resultados: 1) con respecto al THD de la corriente que circula hacia la carga se obtuvo $2.4 \%$ menor a lo establecido que es del $8 \%$ (regulación CONELEC 004/01, NP EN 501602010), 2) con respecto al THD de la tensión medido en el PCC se obtuvo $2.4 \%$ menor a lo establecido, 3) con respecto al THD de la corriente que circula hacia el sistema basado en volante de inercia se obtuvo $3.4 \%$ menor a lo establecido en la normativa.

\section{Conclusiones}

El presente artículo realiza un estudio acerca de los niveles de tensión que se originan con la introducción del sistema de energía basado en volantes de inercia en una red de distribución, ante la presencia de huecos de tensión originados por el ingreso de cargas transitorias a la red eléctrica. De acuerdo con los resultados expuestos se puede concluir que, al momento de cargarse el sistema con la presencia de cargas en la red eléctrica, es regulado el nivel tensión en la red de distribución.

El sistema posee un tiempo de respuesta menor a los milisegundos, por lo tanto, evita la formación de huecos de tensión en el PCC al momento del arranque de cargas importantes conectadas a la red.

Otro aspecto importante es que el sistema de energía basado en volante de inercia no afecta los niveles de THD de la tensión y corriente en el PCC.

Una limitación que presenta este sistema es que una vez llegado a su carga nominal no puede actuar 
ante una sobretensión ( swell), pues ya no va a tener la capacidad de absorber la energía excedente en el PCC, debido a que el nivel de tensión del bus de continua como la velocidad de la máquina síncrona alcanzaron sus valores nominales. De no tomar previsiones, provocaría un deterioro en la vida útil de cada uno de los componentes del sistema, acortando su operatividad en el tiempo.

Un problema que posee este sistema, es que, si el nivel de tensión del bus de continua y la velocidad de la máquina síncrona se ubican por debajo de los valores mínimos permitidos, el sistema ya no va a poder inyectar energía para evitar la formación de los huecos de tensión y si el sistema de control en ese instante actúa de forma errónea, el sistema basado en volante de inercia va a empezar a absorber energía desde la red para ingresar al modo carga involucrando de forma directa una disminución más drástica del nivel de tensión en el PCC. Para solucionar este inconveniente se debe instalar en paralelo otro sistema de inyección de energía auxiliar, el cual debe constar de un sistema de control que monitoree conjuntamente: el nivel de tensión del bus de continua, la velocidad de la máquina síncrona y la tensión de la red de distribución, pues de este modo cuando el sistema basado en volante de inercia y la red de distribución se ubiquen por debajo de los valores permitidos empiece a inyectar energía para evitar lo formación de los huecos de tensión en el PCC.

\section{Agradecimientos}

El primer autor agradece a la Secretaría de Educación Superior, Ciencia, Tecnología e Innovación (Senescyt) de la República del Ecuador por la beca de cuarto nivel.

\section{Referencias}

[1] N. S. Gayathri, N. Senroy, and I. N. Kar, "Smoothing of wind power using flywheel energy storage system," IET Renewable Power Generation, vol. 11, no. 3, pp. 289-298, 2017. [Online]. Available: https://doi.org/10.1049/iet-rpg.2016.0076

[2] D. O. Akinyele and R. K. Rayudu, "Review of energy storage technologies for sustainable power networks," Sustainable Energy Technologies and Assessments, vol. 8, pp. 74-91, 2014. [Online]. Available: https://doi.org/10.1016/j.seta.2014.07.004

[3] S. Gayathri Nair and N. Senroy, "Power smoothening using multi terminal dc based dfig connection and flywheel energy storage system," in 2016 IEEE 6th International Conference on Power Sys- tems (ICPS), 2016, pp. 1-6. [Online]. Available: https://doi.org/10.1109/ICPES.2016.7584134

[4] J. A. Guacaneme, D. Velasco, and C. A. L. Trujillo, "Revisión de las características de sistemas de almacenamiento de energía para aplicaciones en micro redes," Información tecnológica, vol. 25, pp. 175-188, 00 2014. [Online]. Available: http: //dx.doi.org/10.4067/S0718-07642014000200020

[5] D. R. Aitchison, M. Cirrincione, and N. Leijtens, "Design development of a flywheel energy storage system for isolated pacific island communities," in 2016 IEEE International Conference on Advanced Intelligent Mechatronics (AIM), 2016, pp. 1628-1633. [Online]. Available: https://doi.org/10.1109/AIM.2016.7577003

[6] J. Itoh, T. Masuda, D. Sato, T. Nagano, T. Suzuki, and N. Yamada, "Development of magnetic assist system in flywheel energy storage system for power load-leveling," in 2016 IEEE International Conference on Renewable Energy Research and Applications (ICRERA), 2016, pp. 198-203. [Online]. Available: https://doi.org/10.1109/ICRERA.2016.7884537

[7] X. Zhang and J. Yang, "A robust flywheel energy storage system discharge strategy for wide speed range operation," IEEE Transactions on Industrial Electronics, vol. 64, no. 10, pp. 7862-7873, 2017. [Online]. Available: https://doi.org/10.1109/TIE.2017.2694348

[8] F. Franco Hernández and H. Valdés Carrillo, Minería artesanal del oro de aluvión en Mocoa, Putumayo, Amazonia colombiana. Universidad Nacional de Colombia, 2005. [Online]. Available: https://bit.ly/2zTe5uL

[9] G. V. Deshpande and S. S. Sankeshwari, "Speed control of induction motors using hybrid pi plus fuzzy controller," International Journal of Advances in Engineering \& Technology, IJAET, vol. 6, no. 5, pp. 2253-2261, 2013. [Online]. Available: https://bit.ly/3dYCspA

[10] L. G. González Morales, "Mejora de la eficiencia y de las prestaciones dinámicas en procesadores electrónicos de potencia para pequeños aerogeneradores sincrónicos operando en régimen de velocidad variable," Ph.D. dissertation, 2011. [Online]. Available: https://bit.ly/2Tqfksm

[11] F. A. Bardemaker, "Modulação vetorial aplicada a retificadores trifásicos PWM unidirecionais," Ph.D. dissertation, 2006. [Online]. Available: https://bit.ly/3e5gsta 
[12] A. S. de Morais, F. Lessa Tofoli, and I. Barbi, "Modeling, digital control, and implementation of a three-phase four-wire power converter used as a power redistribution device," IEEE Transactions on Industrial Informatics, vol. 12, no. 3, pp. 1035-1042, 2016. [Online]. Available: https://doi.org/10.1109/TII.2016.2544248

[13] S. M. de Pancorbo, G. Ugalde, J. Poza, and A. Egea, "Comparative study between induction motor and synchronous reluctance motor for electrical railway traction applications," in 2015 5th
International Electric Drives Production Conference (EDPC), 2015, pp. 1-5. [Online]. Available: https://doi.org/10.1109/EDPC.2015.7323219

[14] M. Bhardwaj, Sensored Field Oriented Control of 3-Phase Permanent Magnet Synchronous Motors. Texas Instruments, 2013. [Online]. Available: https://bit.ly/3gahvKc

[15] S. Talebi Rafsanjan, "Advanced high-speed flywheel energy storage systems for pulsed power application." Ph.D. dissertation, 2008. [Online]. Available: https://bit.ly/3cPPO7j 